\title{
Proposal of an international harmonized analytical technique for quantifying of residual acetamiprid and imidacloprid in wheat
}

\author{
Naoto Furusawa \\ Graduate School of Human Life Science, Osaka City University, Osaka 558-8585, Japan \\ E-mail: furusawa@life.osaka-cu.ac.jp
}

\begin{abstract}
This paper proposes an international harmonized analytical method for residual monitoring of selected neonicotinoids in crops and presents a fast, easy, and space-saving technique of sample preparation followed by a $100 \%$ water mobile phase high-performance liquid chromatography (HPLC) coupled photo-diode array detector (PDA) for quantifying acetamiprid (ATP) and imidacloprid (ICP) in wheat. The analytes were extracted from the sample using a handheld ultrasonic homogenizer with water, and purified by MonoSpin ${ }^{\circledR} \mathrm{C} 18-\mathrm{CX}$, a centrifugal monolithic silica spin mini-column, and quantified within $20 \mathrm{~min} / \mathrm{sample}$. The accuracy, precision, and system suitability are well within the international method acceptance criteria.
\end{abstract}

Keywords: International Harmonized Analytical Method; Acetamiprid; Imidacloprid; Centrifugal Monolithic Silica Spin Mini-Column.

\section{Introduction}

Some kinds of neonicotinoids, neuro-active/systemic insecticides, began to come under increasing scrutiny over potential environmental impacts, in the early 2000s. The use of neonicotinoids was linked in a range of studies to a number of adverse ecological effects, including honey-bee colony collapse disorder and loss of birds due to reduction in insect populations. Increased scrutiny eventually led to restrictions and bans on the use of different neonicotinoids in several countries (Cressey 2013, European Commission 2013).

In December 2013, two neonicotinoid insecticides, acetamiprid (ATP) and imidacloprid (ICP), may affect the developing human nervous system, disclose the European Food Safety Authority (EFSA). Experts from the Authority propose that some guidance levels for acceptable exposure to the two neonicotinoids be lowered while further research is carried out to provide more reliable data on so-called developmental neurotoxicity (European Food Safety Authority 2013).

Wheat is a very important food because it is grown on more than $216,000,000$ hectares $(530,000,000$ acres) (FAO 2013) larger than for any other crop; its world trade is greater than for all other crops combined; is the world's most favored staple food. The Codex, FAO/WHO Food Standards, has set maximum residue limit (MRL) for the ICP in wheat flour at $0.03 \mathrm{ppm}$ (Codex 2013) to ensure the safety and appropriateness of wheat for human consumption. Monitoring the presence of ATP and ICP in wheat is, therefore, an important means of guaranteeing food safety.

Depending on the recent expansion and diversification in the international food trade, the development of international harmonized methods to determine chemical residues in foods is essential to guarantee equitable international trade in these foods and ensure food safety for consumers. Whether in industrial nations or devel oping countries, an international harmonized method for residue monitoring in foods is urgently -needed. The optimal harmonized method must be easy-to-use, economical in time and cost, and must cause no harm to the environment and analyst.

Although several techniques based on high-performance liquid chromatographic (HPLC) detection have been developed for the monitoring ATP and ICP (Chen et al. 2013, Xiao et al. 2013, Jovanov et al. 2013, Xie et al. 2011, Seccia et al. 2008, Di Muccio et al. 2006, Ferrer et al. 2005), these methods have crucial drawbacks: 1) they involve several analytical steps in the sample preparation, which are time-and cost-consuming and do not permit the determination of large number of samples; 2) all of the methods consume large quantities of toxic organic solvents, acetonitrile and/or methanol (EU classification 1967), in the mobile phases. Risk associated with these solvents extends beyond direct implications for the health of humans and wildlife to affect our environment and the ecosystem in which we all reside. Eliminating the use of toxic solvents and reagents is an important goal in terms of environmental conservation, human health and the economy (Anastas \& Warner 1998, Yoshimura et al. 2001); 3) most of the recent methods are based on LC-MS or -MS/MS. The facilities that LC-MS/MS system is available are limited to part of industrial nations because these are hugely expensive, and the methodologies use complex and specific. These are unavailable in a lot of laboratories for routine analysis, particularly in developing countries. No optimal method that satisfies the aforementioned requirements has yet been identified.

As a technique that can be encouraged as an international harmonized analytical method for the residue monitoring of ATP and ICP, this paper describes a simplified/space-saving sample preparation with minimized organic solvent consumption followed by an isocratic $100 \%$ water mobile phase HPLC for determining ATP and ICP in wheat. 


\section{Materials and methods}

\subsection{Chemicals, reagents, and blank samples}

All chemicals including acetamiprid (ATP) and imidacloprid (ICP) standards were purchased from Wako Pure Chem. Ltd. (Osaka, Japan). Ethanol (non-toxic class, the human or environmental toxicity is negligible [14]) and distilled water were of HPLC grade. Wheat produced by pesticide-free was used as wheat samples for the present study. The wheat was brayed fully (in fine powder) and used as blank wheat samples.

\subsection{Equipment}

The following apparatuses were used in the sample preparation: handheld ultrasonic-homogenizer (model HOM-100, 2 mm ID probe, Iwaki Glass Co., Ltd., Funabashi, Japan); micro-centrifuge (Biofuge ${ }^{\circledR}$ fresco, Kendo Lab. Products, Hanau, Germany); a MonoSpin ${ }^{\circledR}$ as centrifugal monolithic silica spin mini-column (sample throughput volume $\leq 300 \mu \mathrm{L}$ ), MonoSpin C18-CX (bonded with octadecyl group and benzene sulfonic acid group) (GL Sciences, Inc., Tokyo, Japan). A non-polar sorbent columns, Inertsil WP300 C4 $(5 \mu \mathrm{m} \mathrm{d}, 4.6 \times 150 \mathrm{~mm})$ (GL Sciences) for HPLC analysis was used.

The HPLC system, used for method development, included a model PU-980 pump and DG-980-50-degasser (Jasco Corp., Tokyo, Japan) equipped with a model CTO-10AS vp column oven (Shimadzu Scientific Instruments, Kyoto, Japan), as well as a model SPD-M10A VP photodiode-array (PDA) detector (Shimad$\mathrm{zu})$.

\subsection{Operating conditions}

The analytical column was an Inertsil WP300 C4 $(150 \times 4.6 \mathrm{~mm}$, $5 \mu \mathrm{m})$ column using an isocratic mobile phase of water at a flow rate of $1.0 \mathrm{~mL} / \mathrm{min}$ at $50^{\circ} \mathrm{C}$. PDA detector was operated at $190-$ $350 \mathrm{~nm}$ : the monitoring wavelengths were adjusted to 245 and 269 $\mathrm{nm}$ which represent maximums for ATP (at $245 \mathrm{~nm}$ ) and ICP (at $269 \mathrm{~nm}$ ), respectively (Fig. 1). The injection volumes were $10-$ $20 \mu \mathrm{L}$.

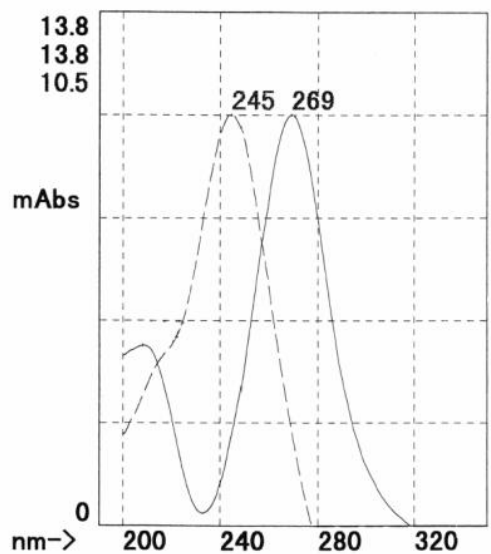

Fig. 1: Typical absorption spectra of peaks for ATP (dashed line, max. 245 $\mathrm{nm}$ ) and ICP (solid line, max. $269 \mathrm{~nm}$ ) standards in the HPLC Chromatogram.

\subsection{Preparations of stock standards and working mixed standard solutions}

Stock standard solutions of ATP and ICP were prepared by dissolving each compound in water followed by water to a concentration of $50 \mu \mathrm{g} / \mathrm{mL}$. Each solution was stored at $-20^{\circ} \mathrm{C}$. Working mixed standard solutions of these two compounds were freshly prepared by suitably diluting the stock solutions with water on the day of the analysis.

\subsection{Preparation of calibration standards and quality control samples}

For method validation studies, calibration standards and quality control samples (QCs), terms defined in the FDA guideline (FDA/CDER/CVM 2001), were prepared by spiking appropriate aliquots of the mixed standard solution in blank wheat samples. Calibration standards were used to construct calibration curves from which the concentrations of analytes in unknown monitoring samples are determined practically. QCs used to evaluate the performance of the proposed method. In this study, the standards were prepared in the range of $0.1-5 \mu \mathrm{g} / \mathrm{g}$ for both analytes. Three QC levels (For both analytes, QC1 $=0.1 \mu \mathrm{g} / \mathrm{g}$; QC2 $=0.5 \mu \mathrm{g} / \mathrm{g}$; $\mathrm{QC} 3=1 \mu \mathrm{g} / \mathrm{g}$ ) were prepared.

\subsection{Sample preparation}

An accurate $0.1 \mathrm{~g}$ sample was taken into a $1.5 \mathrm{~mL}$ microcentrifuge tube and homogenized with $0.6 \mathrm{~mL}$ of water with a handheld ultrasonic-homogenizer for $30 \mathrm{~s}$. After being homogenized, the capped tube was centrifuged at 13,000 rpm for $5 \mathrm{~min}$. A $0.1 \mathrm{~mL}$ of supernatant liquid was poured to a MonoSpin C18-CX and, immediately after, the capped mini-column was centrifuged at 3,500 rpm for $1 \mathrm{~min}$. Under the similar centrifuging operation, the mini-column had been washed with $0.1 \mathrm{~mL}$ of $5 \%$ (v/v) ethanol (in water) and then ATP and ICP was eluted with $0.1 \mathrm{~mL}$ of $30 \%$ ethanol. The eluate was injected into the HPLC system.

\subsection{Method validation}

The performance of the developed method was validated in terms of some parameters from the international guidelines for bioanalytical procedure (FDA/CDER/CVM 2001, FDA 1994, Huber 1998, ICH 1994, AOAC 2012, Codex 2001).

\section{Results and discussion}

\subsection{Sample preparation}

The present procedure is very easy and small-scale technique that minimizes organic solvent consumption in the preparation of analytes. The ultrasonic-homogenization enabled the satisfactory extraction of ATP and ICP from a wheat sample with a $100 \%$ water. The extract obtained by the present operation was purified by subsequent centrifugal monolithic silica spin mini-column, MonoSpin ${ }^{\circledR}$. The spin mini-column is a monolithic SPE column which is said to be excellent for the small volume sample with easy and quick operation by centrifuge (GL Science, http://www.glsciences.com/c-product/sample/solid/monospinseries/). The present procedure can realize a small scale extraction and easy purification of ATP and ICP in a short time while significantly limiting the consumption of organic solvents (an ethanol: non-toxic class) (mere $35 \mu \mathrm{L} /$ sample). The procedure resulted in high recovery and reproducibility.

Fig. 2 illustrates that the resulting chromatograms were free of interfering compounds for the quantification and identification of ATP and ICP by the HPLC, with the PDA detector set at 245 (for ATP) and $269 \mathrm{~nm}$ (for ICP) (giving the maximum absorbance for ATP or ICP). The present HPLC system accomplished good separation with the need for a gradient system to improve the separation and pre-column washing after analysis. 


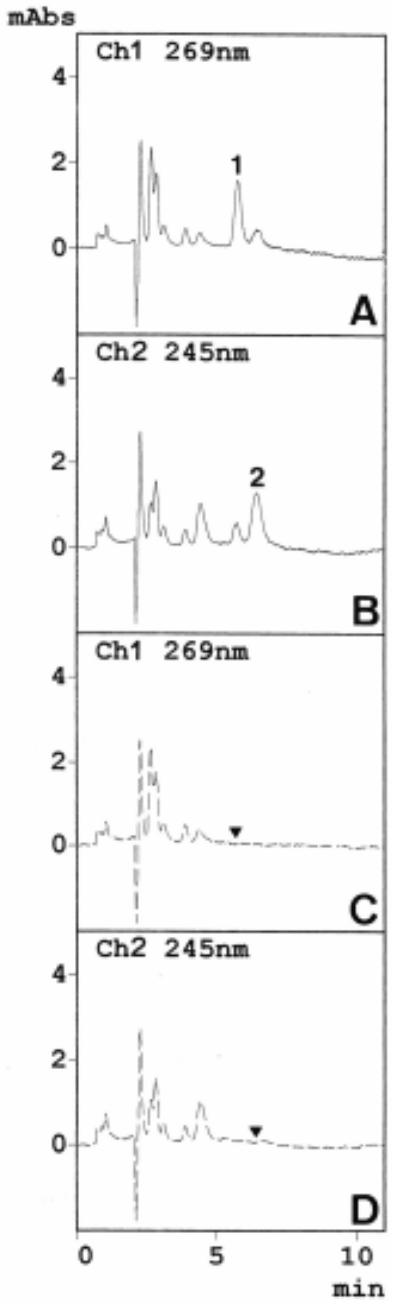

Fig. 2: Chromatograms obtained from the HPLC system for a spiked (each compound $0.5 \mu \mathrm{g} / \mathrm{g}$ ) wheat sample (A, B) and a blank wheat sample (C, D). PDA detector set at $269 \mathrm{~nm}(A, C)$ and $245 \mathrm{~nm}(B, D)$. Peaks, $1=$ ICP (Retention time, $\mathrm{Rt}=5.85 \mathrm{~min}) ; 2=\mathrm{ATP}(\mathrm{Rt}=6.54 \mathrm{~min})$. Closed triangles $(\nabla)$ indicate the retention times of ICP (C) and ATP (D), respectively.

\subsection{Method validation}

\subsubsection{Method validation}

Table 1 summarizes the method validation parameters. The accuracy, precision, and system suitability are within the international method acceptance criteria (FDA/CDER/CVM 2001, FDA 1994, Huber 1998, ICH 1994, AOAC 2012, Codex 2001) (FDA/CDER/CVM 2001, FDA 1994, Huber 1998, ICH 1994, AOAC 2012, Codex 2001).

Table 1: Method Validation Data

\begin{tabular}{lcc}
\hline Parameter & ATP & ICP \\
\hline${\text { Linearity }(\mathrm{r})^{\mathrm{a}}}$ & 0.9948 & 0.9971 \\
Range $(\mu \mathrm{g} / \mathrm{g})$ & \multicolumn{2}{c}{$0.1-5$} \\
Accuracy $^{\mathrm{b}}(\%)$ & 93.8 & 98.0 \\
Precision $^{\mathrm{c}}(\%)$ & 1.8 & 1.6 \\
Sensitivity $^{\mathrm{d}}(\mu \mathrm{g} / \mathrm{g})$ & 0.029 & 0.024 \\
System suitability $^{\mathrm{e}}(\%):$ & & \\
Retention time & 0.11 & 0.07 \\
Peak area & 0.73 & 0.54 \\
\hline
\end{tabular}

${ }^{\mathrm{a}} \mathrm{r}$ is the correlation coefficient $(\mathrm{p}<0.01)$ for calibration curve.

${ }^{\mathrm{b}}$ Average recoveries from 18 replicates (=six replicates at three QC levels $(0.1,0.5$, and $1 \mu \mathrm{g} / \mathrm{g}$ for ATP and ICP, respectively).

${ }^{\mathrm{c}}$ Values are relative standard deviations (RSD, $\mathrm{n}=18$ ).

${ }^{\mathrm{d}}$ Quatitative limit as the concentration of analyte giving a signal-to-noise ratio $=10$.

${ }^{\mathrm{e}}$ Data as the relative standard deviations calculated for 20 replicate injections of the prepared eluate for a rice sample spiked with ATP and ICP (each $0.5 \mu \mathrm{g} / \mathrm{g}$ ).

\subsubsection{Specificity and selectivity}

The application of the proposed procedure to 6 blank wheat samples demonstrated that no interference peak was presented around the retention times for ATP and ICP in any of the sample examined. The present HPLC-PDA system easily confirmed the peak identity of target compound. Both analytes were identified in a wheat sample by their retention times and absorption spectra. The ATP and ICP spectra obtained from the wheat sample were practically identical to those of the standards. Because of the complete separations, PDA detection at trace levels is fully available. It is, therefore, instructive to demonstrate purification effectiveness of the sample preparation. The system did not require the use of MS or MS/MS, which is very expensive and is not available in a lot of laboratories for routine analysis.

\subsubsection{Cost and time performances}

The total time and budget required for the analysis of a single sample was $<20 \mathrm{~min}$ and approximately US $\$ 3.8$ (as of December 11, 2014), respectively. For sequential analysis, a batch of 24 samples could be analyzed in $<4 \mathrm{~h}$. These findings became term required for the routine assay.

\section{Conclusion}

An idiotproof operating sample preparation followed by an isocratic $100 \%$ water mobile phase HPLC-PDA method for simultaneous quantification of ATP and ICP in wheat has been successfully established. The method validation data were well within the international method acceptance criteria. The present procedure provided an easy-to-use, rapid, space-saving, and harmless and resulted in high recovery and repeatability with considerable saving of analysis time/cost. In particular, the present technique may be proposed as an international harmonized method for deterring ATP and ICP in wheat.

\section{References}

[1] Cressey D (2013) "Europe debates risk to bees", Nature 496, 408. http://dx.doi.org/10.1038/496408a.

[2] European Commission, Regulation (EU) No 485/2013, 2013. http://eur-

lex.europa.eu/LexUriServ/LexUriServ.do?uri=OJ:L:2013:139:0012 :0026:EN:PDF.

[3] European Commission, Bees \& Pesticides: Commission goes ahead with plan to better protect bees. 30 May 2013. http://ec.europa.eu/food/archive/animal/liveanimals/bees/neonicoti noids en.htm.

[4] European Food Safety Authority (EFSA), EFSA assesses po-tential link between two neonicotinoids and developmental neurotoxicity. Press release 17 December 2013. http://www.efsa.europa.eu/en/press/news/131217.htm.

[5] FAO, FAOSTAT, Retrieved 23 November 2013 http://faostat3.fao.org/browse/Q/*/E.

[6] Codex Alimentarius, Pesticide Residues in Food and Feed, Codex Pesticides Residues in Food Online Database 2013.

[7] Chen M, et al (2013) Simultaneous determination of residues in pollen and high-fructose corn syrup from eight neonicotinoid insecticides by liquid chromatography-tandem mass spectrometry. Anal. Bioanal. Chem. 405, 9251-9264. http://dx.doi.org/10.1007/s00216013-7338-7.

[8] Xiao Z, et al. (2013) Determination of neonicotinoid insecticides residues in eels using subcritical water extraction and ultraperformance liquid chromatography-tandem mass spectrometry. Anal. Chim. Acta 777, 32-40. http://dx.doi.org/10.1016/j.aca.2013.03.026.

[9] Jovanov P, et al. (2013) Multi-residue method for determination of selected neonicotinoid insecticides in honey using optimized dispersive liquid-liquid microextraction combined with liquid chromatography-tandem mass spectrometry. Talanta 111, 125-133. http://dx.doi.org/10.1016/j.talanta.2013.02.059.

[10] Xie W, et al. (2011) Determination of neonicotinoid pesticides residues in agricultural samples by solid-phase extraction com- 
bined with liquid chromatography-tandem mass spectrometry. J.

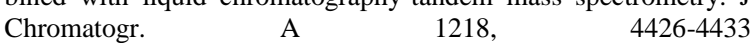
http://dx.doi.org/10.1016/j.chroma.2011.05.026.

[11] Seccia S, et al. (2008) Determination of neonicotinoid insecticides residues in bovine milk samples by solid-phase extraction clean-up and liquid chromatography with diode-array detection. J. $\begin{array}{llll}\text { Chromatogr. } & \text { A } & 1214, & 115-120 .\end{array}$ http://dx.doi.org/10.1016/j.chroma.2008.10.088.

[12] Di Muccio A, et al. (2006) Application of solid-phase extraction and liquid chromatography-mass spectrometry to the determination of neonicotinoid pesticide residues in fruit and vegetables. J. Chromatogr. An 1108, 1-6. http://dx.doi.org/10.1016/j.chroma.2005.12.111.

[13] Ferrer I, et al. (2005) Quantitation and accurate mass analysis of pesticides in vegetables by LC/TOF-MS. Anal. Chem. 77, 28182825. http://dx.doi.org/10.1021/ac048458x.

[14] EU classification (The Dangerous Substances Directive 67/548/EEC): Council Directive 67/548/EEC of 27 June 1967 on the approximation of laws, regulations and administrative provisions relating to the classification, packaging and labelling of dangerous substances.

[15] Anastas, PT, and Warner, JC, Green Chemistry: Theory and Prac tice; Oxford University Press: Oxford, United Kingdom (1998)

[16] Yoshimura, T, et al., Green Chemistry: Aim for the Zero EmissionChemicals, Sankyo Publishing Co. Ltd. Press, Tokyo, Japan (2001).

[17] FDA/CDER/CVM, Guidelines for Industry - Bioanalytical Method Validation, 2001. http://www.fda.gov/downloads/Drugs/GuidanceComplianceRegula toryInformation/Guidances/UCM070107.pdf.

[18] FDA, Reviewer Guide, Validation of Chromatographic Method Center Drug Evaluation and Research (CFDER), Silver Spring, MD, USA, 1994.

[19] Huber, L, Validation and Quantification in Analytical Laboratories, Interpharm Press, East England, CO, USA, 1998.

[20] ICH, Work Products, ICH Guidelines, Quality Guidelines- Validation of analytical procedures: Test and methodology Q2 (R1), 1994. http://www.ich.org/products/guidelines.html.

[21] AOAC International, Guidelines for Standard Method Performance Requirements, 2012. http://www.eoma.aoac.org/app f.pdf.

[22] Codex alimentarius commission, ALINORM 01/23, Codex Committee on Methods of Analysis and Sampling, Joint FAO/WHO Food Standards Programme, the 23nd Session of the Codex Committee on Method of Analysis and Sampling, March 2001.

[23] GL Sciences, Monolithic SPE Column for the Purification and Enrichment of Small Amount Sample, MonoSpin ${ }^{\circledR}$ Series. http://www.glsciences.com/c-product/sample/solid/monospinseries/. 